\title{
Differential Incidence of Eyelid Margin Cysts in ASU Outpatients
}

\author{
Mayar Mohammad Salaheldin Faheem, Sherif Elwan, Azza Mohamed Ahmed Said, Ossama Tarek \\ Nada
}

Department of Ophthalmology, Faculty of Medicine, Ain Shams University

Corresponding author: Mayar Mohammad Salaheldin Faheem, E-Mail: Mayar.qassem@ gmail.com, Mobile: +2 01097316366

\begin{abstract}
Background: Wide varieties of lesions affecting the eyelid are encountered within routine Ophthalmology practice. These lesions are numerous due to the unique anatomical features of the eyelid as the whole skin structures, appendages, muscle, modified glands, and conjunctival mucous membrane are represented in the eyelid. The eyelid comprises different types of glands that clinically correlate with the development of eyelid cysts; Sebaceous glands (Meibomian glands and glands of Zeis), aporcine glands (glands of Moll) and eccrine sweat glands.

Objectives: To report the incidence of the different etiologies of eyelid margin cysts in Ain Shams University (ASU) Hospital, General Ophthalmology Outpatients Clinic.

Patients and Methods: It included all patients who presented to the General Ophthalmology Outpatient Clinic, 6 days per week for one month (November 2017) complaining of eyelid margin lesions, found cystic on slit lamp examination, of all ages.

Results: The total number of patients recorded was 45 patients presenting with eyelid margin cysts, out of 1920 patients visiting the General Ophthalmology Outpatient Clinic during the month of November 2017 with an incidence of $2.34 \%$. Seven types of cysts were found namely; External Hordeolum (8.9\%), Internal Hordeolum (15.6\%), Chalazion (28.9\%), Sebaceous Cyst (11.1\%), Cyst of Zeis Gland (13.3\%), Apocrine Hidrocystoma (11.1\%) and Eccrine Hidrocystoma (11.1\%).

Conclusion: Eyelid margin cysts should be studied and categorized as a separate entity from eyelid lesion due to the unique anatomy of the eyelid margin. The incidence was calculated to be $2.34 \%$ during this study that was relatively short lived. Chalazion was the most common lesion encountered but that was solely by clinical diagnosis.

Recommendations: It is recommended to continue this study for a longer duration to be more comparable with the other studies and it should be enriched by histopathological diagnosis aside the clinical one.
\end{abstract}

Keywords: Eyelid, Margin, Cysts

\section{INTRODUCTION}

Wide varieties of lesions affecting the eyelid are encountered within routine Ophthalmology practice. These lesions are numerous due to the unique anatomical features of the eyelid as the whole skin structures, appendages, muscle, modified glands, and conjunctival mucous membrane are represented in the eyelid ${ }^{(1)}$.

The eyelid is composed of two lamellae: the anterior lamella, composed of skin and orbicularis oculi muscle, and the posterior lamella, composed of the tarsal plate and conjunctiva ${ }^{(2)}$.

The lid margin is about $2 \mathrm{~mm}$ thick, horizontally placed, and has a sharp posterior border and a round anterior border. The round anterior border has hair follicles of the eyelashes, into which the sebaceous gland of Zeis and the sweat gland of Moll open. Just anterior to the sharp posterior border of the lid margin is the opening of the tarsal Meibomian gland. Just anterior to the opening of the Meibomian gland is a gray line along which the lid can be vertically split into its anterior soft skin- muscle layer and the hard posterior tarso-conjunctival layer by a knife ${ }^{(3)}$.

The eyelid comprises different types of glands that clinically correlate with the development of eyelid cysts; Sebaceous glands (Meibomian glands and glands of Zeis), aporcine glands (glands of Moll) and eccrine sweat glands ${ }^{(4)}$.

The tarsal glands of Meibom (glandulae tarsales) are large sebaceous glands located in the eyelids and, unlike those of the skin, are unassociated with hairs. These separate glands are arranged in parallel in a single row throughout the length of the tarsal plates in the upper and lower lids, and they presumably act in a coordinated fashion that is influenced by hormonal and neural regulation and by the mechanical forces of muscle contraction during the eye blink ${ }^{(5)}$. They produce the lipid that lines the layer of tear film ${ }^{(6)}$.

The human glands of Moll are specialized apocrine sweat glands located at the margin of the eyelids near the lash follicles. The nature and function of the secretions of the glands of Moll is unknown ${ }^{(7)}$, however, it likely includes the production and 
secretion of antimicrobial components, such as betadefensins and cathelicidin ${ }^{(6)}$.

The eye lashes often contain sebaceous glands (known as Zeis glands) that emanate from the eyelid immediately adjacent to the apocrine glands of Moll. Their lipid rich secretions coat the eyelashes ${ }^{(6)}$.

Various types of cysts can be identified in the eye lid, for example:

Chalazion, Cyst of Zeis Gland, Cyst of Moll Gland, Sebaceous cyst, Comedones, Epidermal inclusion cyst, Epidermal cyst, Dermoid cyst, Eccrine hidrocystoma ${ }^{(8)}$.

\section{AIM OF THE WORK}

To report the incidence of the different etiologies of eyelid margin cysts in Ain Shams University (ASU) Hospital, General Ophthalmology Outpatients Clinic.

\section{PATIENTS AND METHODS}

This is an observational study, conducted at the General Ophthalmology Outpatient Clinic, Ophthalmology Department, Ain Shams University, using non-probability (convenience) sample.

It included all patients who presented to the General Ophthalmology Outpatient Clinic, 6 days per week for one month (November 2017) complaining of eyelid margin lesions, found cystic on slit lamp examination, of all ages.

Patients who had solid masses, cysts with ulcers or undergone previous eyelid surgery were excluded from the study.

The study was conducted in accordance with the ethical standards stated by the Ethical Committee of Ain Shams University, Faculty of Medicine. An informed consent was taken from patients to have them anonymously photographed.

All patients underwent the following: Detailed History was taken from the patients including age, medical history, residence, duration of the lesion and history of recurrence. Ophthalmological examination in the form of Slit lamp biomicroscopy to examine the eyelids. Transillumination test was performed to differentiate translucent and opaque lesions. Anonymous photography using a smart phone 13megapixel camera.
The collected data were coded, tabulated, and statistically analyzed using SPSS 13 software (SPSS Inc., Chicago, Illinois, USA) and Microsoft Excel Windows 2016. Description of qualitative variables was in the form of numbers and percentages and quantitative data was presented as mean and standard deviation.

\section{RESULTS}

The total number of patients recorded was 45 patients presenting with eyelid margin cysts, out of 1920 patients visiting the General Ophthalmology Outpatient Clinic during the month of November 2017 with an incidence of $2.34 \%$.

Seven types of cysts were found namely; External Hordeolum (8.9\%), Internal Hordeolum (15.6\%), Chalazion (28.9\%), Sebaceous Cyst (11.1\%), Cyst of Zeis Gland (13.3\%), Apocrine Hidrocystoma (11.1\%) and Eccrine Hidrocystoma $(11.1 \%)$. Graph.1 shows various types of cysts encountered during this study.

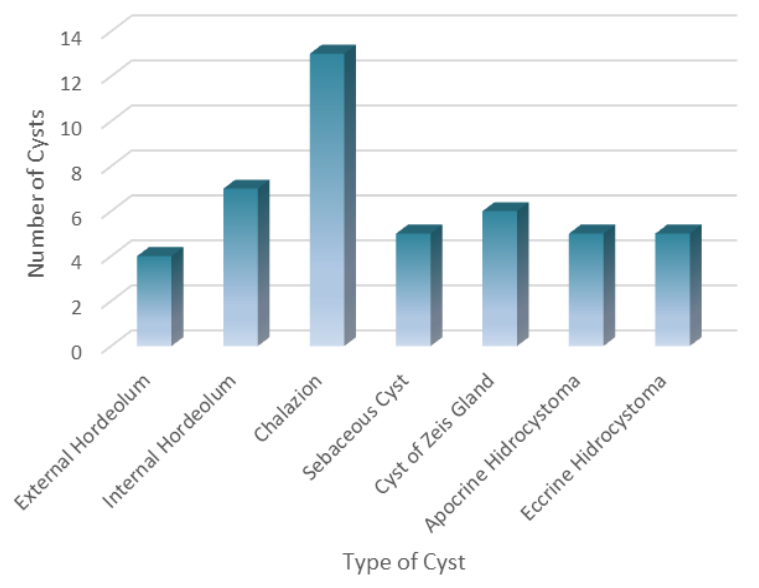

Graph (1): Predilection of Each Type of Cyst

The mean age calculated among the study group was 40.2 years $\pm 20.06 \mathrm{SD}$. Table 1 shows the distribution of cysts among different age groups.

Table (1): Distribution of Cysts Among Different Age Groups.

\begin{tabular}{|c|c|c|c|c|c|c|c|c|}
\hline $\begin{array}{c}\text { Age } \\
\text { (years) }\end{array}$ & $\begin{array}{c}\text { External } \\
\text { Hordeolum }\end{array}$ & Hordeolum & Chalazion & $\begin{array}{c}\text { Sebaceous } \\
\text { Cyst }\end{array}$ & $\begin{array}{c}\text { Cyst of } \\
\text { Zeis } \\
\text { Gland }\end{array}$ & $\begin{array}{c}\text { Apocrine } \\
\text { Hidrocystoma }\end{array}$ & $\begin{array}{c}\text { Eccrine } \\
\text { Hidrocystoma }\end{array}$ & Total \\
\hline $0-20$ & $2(28.5 \%)$ & $3(42.8 \%)$ & $3(42.8 \%)$ & $1(14.2 \%)$ & 0 & 0 & 0 & 7 \\
\hline $21-40$ & $2(13.3 \%)$ & $3(20 \%)$ & $6(40 \%)$ & 0 & $2(13.3 \%)$ & 0 & $1(6.6 \%)$ & 15 \\
\hline $41-60$ & 0 & 0 & $4(25 \%)$ & $3(18.7 \%)$ & $3(18.7 \%)$ & $2(12.5 \%)$ & $3(18.7 \%)$ & 16 \\
\hline $61-80$ & 0 & $1(16.6 \%)$ & 0 & $1(16.6 \%)$ & $1(16.6 \%)$ & $2(33.3 \%)$ & $1(16.6 \%)$ & 6 \\
\hline$>80$ & 0 & 0 & 0 & 0 & 0 & $1(100 \%)$ & 0 & 1 \\
\hline Total & 4 & 7 & 13 & 5 & 6 & 5 & 5 & \\
\hline
\end{tabular}


From this table, the most common age group affected was 21-60 years and chalazion was the most common cyst in this age group.

Sixteen lesions presented in males while twenty-nine lesions presented in females (Graph 2).

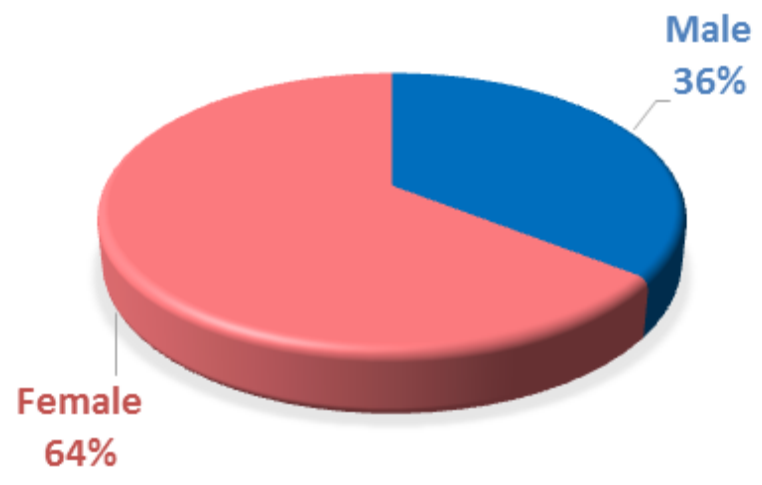

Graph (2): Sex Predilection of Eyelid Margin Cysts.

Sixteen Lesions (35.6\%) were found in the upper eyelid, twenty-four lesions (53.3\%) were found in the lower eyelid and only five lesions $(11.1 \%)$ were found in both the upper and lower.

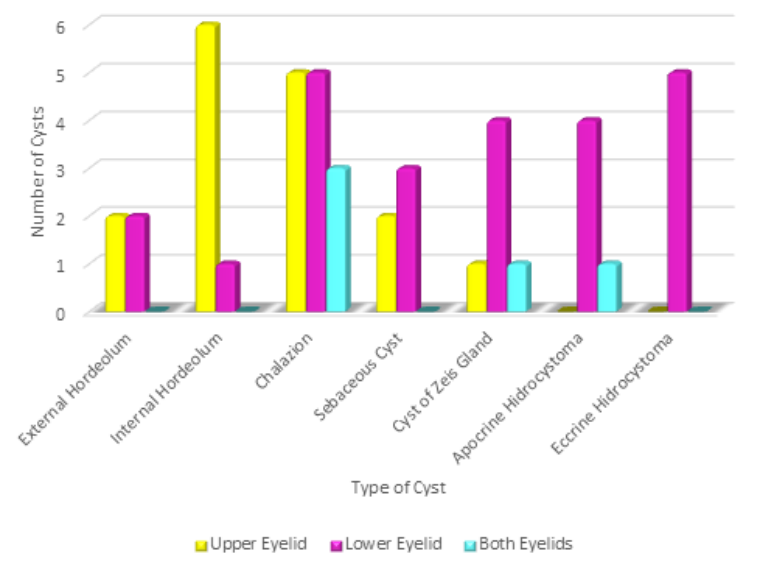

Graph (3): Distribution of Eyelid Margin Cysts according to Site.

\section{DISCUSSION}

To our knowledge this is the first study conducted to measure the incidence of eyelid margin cysts specifically and to calculate the differential incidence of each type of cyst on clinical basis only, in contrast to other retrospective studies that described the incidence of eyelid masses on clinical and histopathological basis.

The incidence of eyelid margin cysts calculated was $2.34 \%$ of the whole study population, in contrast to the $11 \%$ and $20 \%$ of benign eyelid lesions calculated by Al-Feky $\boldsymbol{e t}$ al . (1) and Asproudis et al. ${ }^{(9)}$ respectively. This can be accounted to the relatively shorter period of our study (1 month) in comparison to the 5 and 10-year data (respectively) that were collected retrospectively.

The most common type of cyst encountered in the present study was chalazion (28.9\%) followed by sweat gland hidrocystoma (22.2\%), these results are comparable with the results of Al-Feky $\boldsymbol{e t}$ al. ${ }^{(1)}$ that specified sweat gland hidrocystoma with $29.3 \%$ followed by chalazion with $21.6 \%$. This can be explained by the climatic change between Egypt and Saudi Arabia, the climate in the latter being more humid enhancing the development of sweat gland hidrocystomas more.

In most of the studies found in literature, females are more affected by eyelid lesions than males. The same can be said about the current study that described eyelid margin cysts more in females with $64 \%$. Same can be said about the age group most affected by benign eyelid lesions being around the mid-forties in all the previously mentioned studies, similarly the present study calculated the mean age of patients to be 40.2 years \pm 20.06 SD.

There is no previous mention in literature about whether there is an effect of residence and occupation on the incidence of eyelid margin cysts. The current study calculated an increased incidence of eyelid margin cysts among Cairo city residence (73.3\%) but this cannot be accurate because of immigration from rural (upper and lower Egypt) to urban cities (Cairo). Also, the effect of occupation on incidence of eyelid margin cysts cannot be proven as most patients in the present study were unemployed mainly house wives.

Regarding the site of lesion, this study described lesions affecting the lower lid (53.3\%) more than the upper lid and both lids $(35.6 \%$ and $11.1 \%$ respectively). On the contrary, Bagheri et al. (10) who stated that; the most common site of involvement was the upper lid (58\%) followed by the lower lid (29\%).

It was noted that recurrence and multiplicity of lesions were commonest with patient complaining of chalazion with $30 \%$ of lesions being recurrent and and $23 \%$ of lesions 
being multiple. This can be accounted to that chalazion is great masquerader and can very commonly be a misdiagnosis. As mentioned before intrakeratinous cyst is a rising new entity that must always be kept in mind in cases of recurrent chalazia that can only be differentiated from chalazion by histopathology. Al-Feky et al. ${ }^{(I)}$ clinically diagnosed 48 lesions $(21.6 \%)$ as chalazia, 4 of whiche were proved to be otherwise by histopathology.

\section{CONCLUSION AND RECOMMENDATION}

Eyelid margin cysts should be studied and categorized as a separate entity from eyelid lesion due to the unique anatomy of the eyelid margin. The incidence was found to be $2.34 \%$ during this study that was relatively short lived. Chalazion was the most common lesion encountered but that was solely by clinical diagnosis.

It is recommended to continue this study for a longer duration to be more comparable with other mentioned studies and it should be enriched by histopathological diagnosis aside the clinical one.

\section{REFERENCES}

1- Al-Faky YH (2012): Epidemiology of benign eyelid lesions in patients presenting to a teaching hospital. Saudi J of Ophthalmol., 26:211-216.

2- Chang EI, Esmaeli B, Butler CE (2017): Eyelid reconstruction. Plast Reconstr Surg., 2017; 140:725-735.

3- Ansari MW, Nadeem A (2016): Atlas of Ocular Anatomy. Illinois: Springer,Chapter 5, PP: 53-54.

4- Bhavya PM, Letha V (2017): Profile of eye lid lesions over a decade: a histopathological study from a tertiary care center in South India. Int J Adv Med., 4:1406-1411.

5- Knop E, Knop N, Millar T, Obata H, Sullivan DA (2011): The International Workshop on Meibomian Gland Dysfunction: Report of the Subcommittee on Anatomy, Physiology, and Pathophysiology of the Meibomian Gland. Invest Ophthalmol Vis Sci., 52: 1938-1978.

6- Paus R, Burgoa I, Platt CI, Griffiths T, Poblet E, Izeta A (2016): Biology of the eyelash hair follicle: an enigma in plain sight. Br J Dermatol., 174:741-752.
7- Stoeckelhuber BM, Welsch U, Stoeckelhuber M (2003): Human Glands of Moll: Histochemical and Ultrastructural Characterization of the Glands of Moll in the Human Eyelid. J Invest Dermatol., 121: 28-36.

8- Bowling B (2016): Kanski's Clinical Ophthalmology: A systemic approach $\left(8^{\text {th }}\right.$ Ed.) Sydney: Elservier, Chapter 1, PP: 2-6.

9- Bagheri A, Tavakoli M, Kanaani A, Zavareh RB, Esfandiari H, Aletaha M, Salour H (2013): Eyelid Masses: A 10-year Survey of a Tertiary Hospital in Tehran. Middle East Afr J Ophthalmol., 20: 187-192.

10- Asproudis I, Sotiropoulos G, Gartzios C, Raggos V, Papoudou-Bai A, Katsanos A, Tatsioni A (2015): Eyelid Tumors at the University Eye Clinic of Ioannina,Greece: A 30-year Retrospective Study. Middle East Afr J Ophthalmol., 22: 230-232. 